Filozofska fakulteta, Univerza v Ljubljani

urska.valencicarh@ff.uni-lj.si

\title{
BERNSTEIN, NILS: „KENNEN SIE MICH HERREN/MEINE DAMEN UND HERREN". PHRASEOLOGISMEN IN MODERNER LYRIK AM BEISPIEL VON ERNST JANDL UND NICANOR PARRA.
}

Bernstein, Nils (2011): ,, kennen sie mich herren/meine damen und herren”. Phraseologismen in Moderner Lyrik am Beispiel von Ernst Jandl und Nicanor Parra. Würzburg: Königshausen\&Neumann. ISBN: 978-3-8260-4699-5, mehka vezava, 262 strani, 38,00 EUR

Im Mittelpunkt der Dissertation von Nils Bernstein steht die Phraseologie in der modernen Lyrik des österreichischen Autors Ernst Jandl und des chilenischen Autors Nicanor Parra unter literaturwissenschaftlichen und poetologischen Aspekten. Seine Arbeit ist ein Beitrag zur Erforschung der Rolle von Phrasemen in moderner Lyrik.

Nicanor Parra und Ernst Jandl gelten als zwei zentrale Vertreter moderner Lyrik der zweiten Hälfte des zwanzigsten Jahrhunderts in der jeweiligen Literatur ihres Kulturraumes, die Affinitäten zur Avantgarde und zur klassischen Moderne aufweisen. Beide Dichter brechen mit Normen und zielen auf Entautomatisierung eingefahrener Wahrnehmungsweisen.

Unter Phrasemen werden hier graduell feste Mehrwortverbindungen, die teilweise semantisch transformiert wurden, verstanden. Bernstein bevorzugt den Terminus Phraseologismus zugunsten des Begriffes Phrasem, der sich aber wegen der leichteren internationalen Verwendbarkeit langsam durchzusetzen scheint. Der Gebrauch von Phraseologie wird auf innovative Verfahren lyrischer Produktion hin untersucht. Beide Dichter zeichnet nicht nur hohe Sprachsensibilität, sondern auch ein innovativer Umgang mit dem phraseologischen Sprachmaterial aus. Im Gegensatz zu den existierenden Interpretationsansätzen, die das Phraseologische bei Parra als ein charakteristisches Merkmal und die bei Jandl oft vorkommenden verfremdeten Mehrwortverbindungen als Wortspiele nicht weiter differenzieren, hat die zu rezensierende Arbeit zum Ziel, allgemeingültige Funktionshypothesen über die Verwendung der Phraseme in moderner Lyrik abzuleiten und damit Sprach- und Literaturwissenschaft zusammenzuführen. Durch eine phraseologisch geschulte Lesart wird ein interpretationserweiternder Zugang zu moderner Lyrik mit heuristischem Interesse verschafft. 
Nach Vorstellung der Fragestellung und der Forschung über Phraseme in der Literatur sowie bei Jandl und Parra im Kap. 1 folgt ein Überblick über den Stand der phraseologischen Forschung. Dabei geht es zunächst um die Fragen der Terminologie und schließlich der Klassifikation von Phrasemen im Kap. 2. Poetologische Konzepte Jandls „I'm not / a concrete pot“ und Parras ,Antipoesia“ sowie Verknüpfungspunkte zwischen zwei Dichtern aus unterschiedlichen Kulturräumen, dem lateinamerikanischen und dem westeuropäischen, die in bibliographischer als auch werkkonzeptioneller Hinsicht nicht zu übersehen sind, sind die weiteren Themen im Kap. 3.

Im Kap. 4 über Wirkungsweisen von Phrasemen in der Lyrik von Jandl und Parra zeigt Nils Bernstein am phraseologischen Sprachmaterial deutlich und anschaulich, wie kontextuelle Verfremdung, Erweiterung und Remotivierung ein sprachkritisches Konzept reflektieren. In den beiden Werken stellt der Autor exemplarisch sieben Wirkungsweisen von Phrasemen fest. Die analysierte themenentfaltende Funktion (Kap. 4.1) veranschaulichte, welchen Reiz Phraseme auf die Autoren ausüben, sodass einzelne Strophen oder ganze Gedichte thematisch von der wörtlichen oder übertragenen Lesart eines Phrasems bestimmt sind. Mittels kommunikativer Phraseme wie Routineformeln (Kap. 4.2) verstoßen die beiden Autoren gegen Normen der Lyrik, wenn z. B. ein Gedicht mit einem für die Ansprache üblichen kommunikativen Phrasem beginnt oder endet. Der Bruch mit lyrischen Konventionen durch den gehäuften Gebrauch von Routineformeln steht laut Bernstein im Zusammenhang mit der Herstellung von Unmittelbarkeit und mündlicher Authentizität durch Phraseme und dient als Annäherung an die Alltagssprache (Kap. 4.3). Die beiden Autoren gleichen sich in der Arbeitsweise, indem sie pragmatisch feste Phraseme in einem neuen Kontext verwenden. Bei Parra dient die Annäherung an die Alltagssprache in erster Linie dem Bruch mit Erwartungshaltungen. Im Kap. 4.4 stellt Bernstein fest, dass Geflügelte Worte religiösen Ursprungs bei den beiden untersuchten Autoren Mittel für die autobiographische Imagination darstellen und in die eigene familiäre Sozialisation eingearbeitet werden. Wenn Phraseme auf ihren Wortinhalt geprüft und remotiviert werden, wird in Parras und Jandls Werken die sprachkritische Methode (Kap. 4.5) demonstriert. Sehr häufig dienen modifizierte Phraseme den vielfältigen Ausformungen des Komischen (Kap.4.6). Die Frage nach phraseologisierungsfähigen Wendungen im Werk der beiden Autoren (Kap. 4.7) beantwortet Bernstein mit der Aufzählung von vier Eigenschaften, die Phraseologisierung beeinflussen: eine breite Rezeption, die Bildhaftigkeit zur Gewährleistung einer geringeren Opazität und erhöhten Motiviertheit, die Gestaltung nach rhetorischen Mustern zur Gewährleistung der Memorierbarkeit sowie die Treffsicherheit und Originalität einer Formulierung, die einen Sachverhalt in konziser Weise ausdrückt. Außer des ersten Kriteriums lassen sich aus der Lyrik von Jandl und Parra zwei zentrale Beispiele finden, für die die oben aufgezählten Kriterien belegbar sind: Jandls Gedicht lichtung (Behauptung: »lechts und rinks kann man nicht verwechsern«) und Parras Artefacto (Modifikation vom Geflügelten Wort: »La izquierda y la 
derecha unidas/ jamás serán vencidas«). Im Kap. 5 folgt eine detaillierte Zusammenfassung mit Ausblick auf weitere wünschenswerte Untersuchungen zu Jandl und Parra. Im Abbildungsverzeichnis im Kap.6 werden fünf Abbildungen hinzugefügt. Das Literaturverzeichnis umfasst 35 Seiten.

Eine große Stärke der Interpretation von Nils Bernstein besteht darin, Erkenntnisse der Phraseologie praktisch, jedoch hochsensibel mit den Poetizitätskonzepten moderner Lyrik systematisch zu verbinden. Es lohnt sich, die Interpretation Bernsteins aufzunehmen und sie an vielen anderen literarischen Werken anzuwenden, aber nicht nur, weil Untersuchungen zum Gebrauch von Phrasemen in moderner Literatur, vor allem in moderner Lyrik, zu den Desiderata der Phraseologieforschung gehören, sondern deshalb, weil das Buch eine Einladung zur Eröffnung weiterer literaturwissenschaftlich relevanter Fragestellungen durch die Augen der phraseologisch geschulten Lesart darstellt. 